\title{
Blockchain based secure data handover scheme in non-orthogonal multiple access
}

\author{
Anik Islam*, Mohammed Belal Uddin ${ }^{\dagger}$, Md. Fazlul Kader ${ }^{\ddagger}$ and Soo Young Shin ${ }^{\S}$ \\ ${ }^{*} \dagger$ Wireless and Emerging Networks System (WENS) Laboratory, Department of IT Convergence Engineering, \\ Kumoh National Institute of Technology (KIT), Gumi, South Korea \\ ${ }^{\ddagger}$ Department of Electrical and Electronic Engineering, University of Chittagong, Chittagong, Bangladesh \\ Email: $\left\{{ }^{*}\right.$ anik.islam, ${ }^{\dagger}$ ahad.belal, ${ }^{\S}$ wdragon $\} @$ kumoh.ac.kr, ${ }^{\ddagger}$ f.kader@cu.ac.bd
}

\begin{abstract}
Non-orthogonal multiple access (NOMA) with successive interference cancellation receiver is considered as one of the most potent multiple access techniques to be adopted in future wireless communication networks. Data security in the NOMA transmission scheme is on much attention drawing issue. Blockchain is a distributed peer-to-peer network enables a way of protecting information from unauthorized access, tempering etc. By utilizing encryption techniques of blockchain, a secured data communication scheme using blockchain in NOMA is proposed in this paper. A two-phase encryption technique with key generation using different parameter is proposed. In the first-phase data is encrypted by imposing users' public key and in the second phase, a private key of the base station (BS) is engaged for encryption. Finally, the superiority of the proposed scheme over existing scheme is proven through a comparative study based on the different features.
\end{abstract}

Index Terms-Blockchain, data security, next generation wireless communication, non-orthogonal multiple access, successive interference cancellation

\section{INTRODUCTION}

$\mathbf{T}$ HE provision of high data rate and facilitation of multiple users to communicate simultaneously within a core network is very crucial to meet the quality of service requirement in the era of evolving wireless communication technologies. Simultaneous information exchange among a large number of devices by exploiting limited bandwidth is another challenge of future wireless communication. To overcome the problems and meet the challenges of upcoming wireless networks, nonorthogonal multiple access (NOMA) technique with successive interference cancellation (SIC) receiver is considered as one of the most promising multiple access techniques [1]-[4]. In NOMA, multiple users are facilitated to transmit/receive data simultaneously at the same frequency by using power division multiplexing. In downlink NOMA, a base station (BS) transmits superposed data for the intended users by allocating more power to the weaker user $\mathrm{UE}_{2}$ (User Equipment) data than stronger user $\mathrm{UE}_{1}$ data, as shown in Fig. $1 \mathrm{UE}_{1}$ first decodes data bits of $\mathrm{UE}_{2}$. After reconstructing the signal related to those decoded data bits, it's been cancelled from the total received signal. This traditional successive interference cancellation continues till the decoding of $\mathrm{UE}_{1}$ 's data bits.

As described earlier, due to the way of traditional SIC procedure, the data of the weak user is not secured to the

978-1-5386-6163-5/18/\$31.00 @2018 IEEE

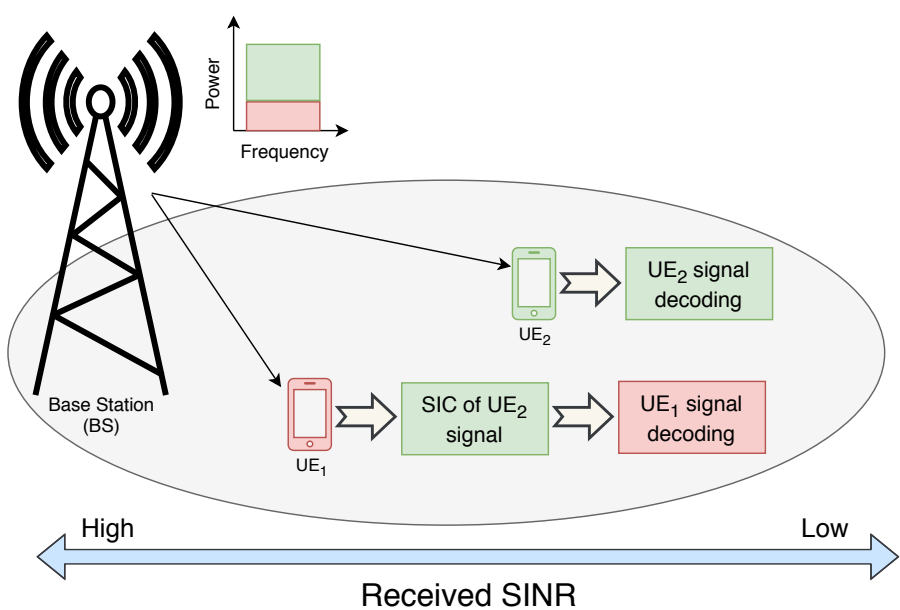

Fig. 1. Basic downlink NOMA with SIC.

strong user. Moreover, most of the existing NOMA-based systems mainly focus on traditional SIC [1]-[6]. That is why proper security is needed during SIC to prevent leakage of weak users' data information to the strong user.

A secure way of performing SIC for NOMA was discussed in [7]. In [7], they have shown similar concern about aforementioned security issues. In order to solve that, they have adopted symmetric key encryption and they have generated the key based on international mobile equipment identity (IMEI), media access control (MAC) address. Using IMEI and MAC address could pose vulnerability towards spoofing attack. They have tried to solve it using "sticky MAC address". But this technique is used only by the vendors named CISCO and Juniper. So, this could not give solutions to the aforementioned security issues for all users.

Blockchain has recently gained tremendous attention due to its promise of ensuring security of data. Blockchain is a data structure which is shared and replicated among the participant of the network. Blockchain was first introduced by Satoshi Nakamoto with bitcoin [8]. In blockchain, a pair of keys (private/public) is adopted. The public key is used as an identity for the user so that users' privacy can remain concealed and the private key is used for encrypting data so that data can remain protected [9], [10]. However, this privacy 


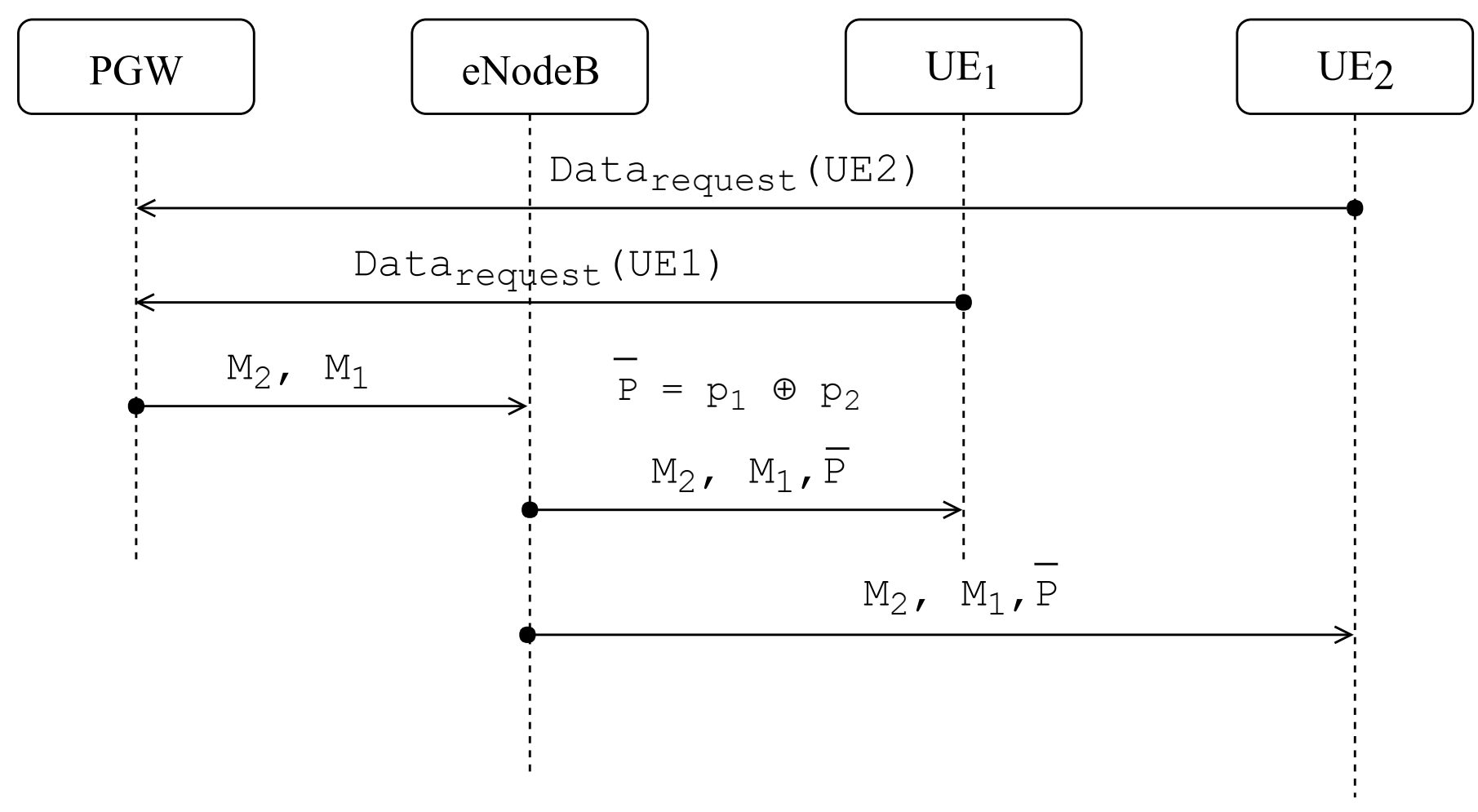

Fig. 2. Existing data handover scheme [7].

protection technique can mitigate the issue of disclosing data to the strongest user.

In this paper, a blockchain based secure data handover technique in NOMA is proposed in order to mitigate the mentioned above-mentioned security issues. The contribution of this paper is outlined below:

- A key generation technique is proposed using different parameter.

- A two-phase encryption is proposed so that data can be protected from any kind of attacks.

The remaining sections of this paper are organized as follows: Section II illustrates the existing data handover scheme. The proposed blockchain based data handover scheme is portrayed in Section IIII A performance comparison between proposed scheme and [7] is demonstrated in Section IV] Finally, Section $\mathrm{V}$ draws a conclusion from this paper with future research directions.

\section{EXISTING DATA HANDOVER SCHEME}

Existing data handover scheme contains UEs, eNodeB (Evolved Node-B), and PGW (Packet gateway), as shown in Fig. 2 All the notations and their description are provided in Table II Fig. 2 illustrates data handover scheme assuming two UEs, one BS, one PGW and the scheme is considered for downlink transmission. The procedure of existing handover applying SIC is depicted below [7]:

1) Both $\mathrm{UE}_{1}$ and $\mathrm{UE}_{2}$ send data request to eNodeB which eNodeB forwards to PGW.
TABLE I

NOTATIONS AND THEIR DESCRIPTION

\begin{tabular}{c|l}
\hline Notation & Description \\
\hline \hline$P K_{\mathrm{UE}_{t h}}$ & Public key for $\mathrm{UE}_{t h}$ \\
$P R_{\mathrm{UE}_{t h}}$ & Private key for $\mathrm{UE}_{t h}$ \\
$P R_{B}$ & Private key for BS \\
$Z_{B D}$ & Blockchain based database \\
$p_{t h}$ & Allocated power for UE \\
$\bar{P}$ & Total allocated power \\
$M_{t h}$ & Signals for UE \\
$E_{P K}()$. & Encryption using public key \\
$E_{P R}()$. & Encryption using private key \\
$D_{P K}()$. & Decryption using public key \\
$D_{P R}()$. & Decryption using private key \\
$I M$ & IMEI \\
$M$ & MAC address \\
$T$ & Timestamp \\
$L a t$ & Latitude \\
$L o n$ & Longitude \\
\hline
\end{tabular}

2) PGW picks unencrypted $M_{2}$ and $M_{1}$ for $\mathrm{UE}_{2}$ and $\mathrm{UE}_{1}$ respectively, and forwards to eNodeB.

3) After getting $M_{2}$ and $M_{1}$ from PGW, eNodeB allocates power $p_{1}$ and $p_{2}$ with signals of $\mathrm{UE}_{2}$ and $\mathrm{UE}_{1}$ respectively, and superposes both signals to $\bar{P}=p_{1} \oplus p_{2}$. eNodeB returns $M_{2}$ and $M_{1}$ with $\bar{P}$ back to $\mathrm{UE}_{2}$ and $\mathrm{UE}_{1}$.

4) When $\mathrm{UE}_{1}$ receives data from eNodeB, it decodes all data bits subsequently and after decoding, it subtracts 


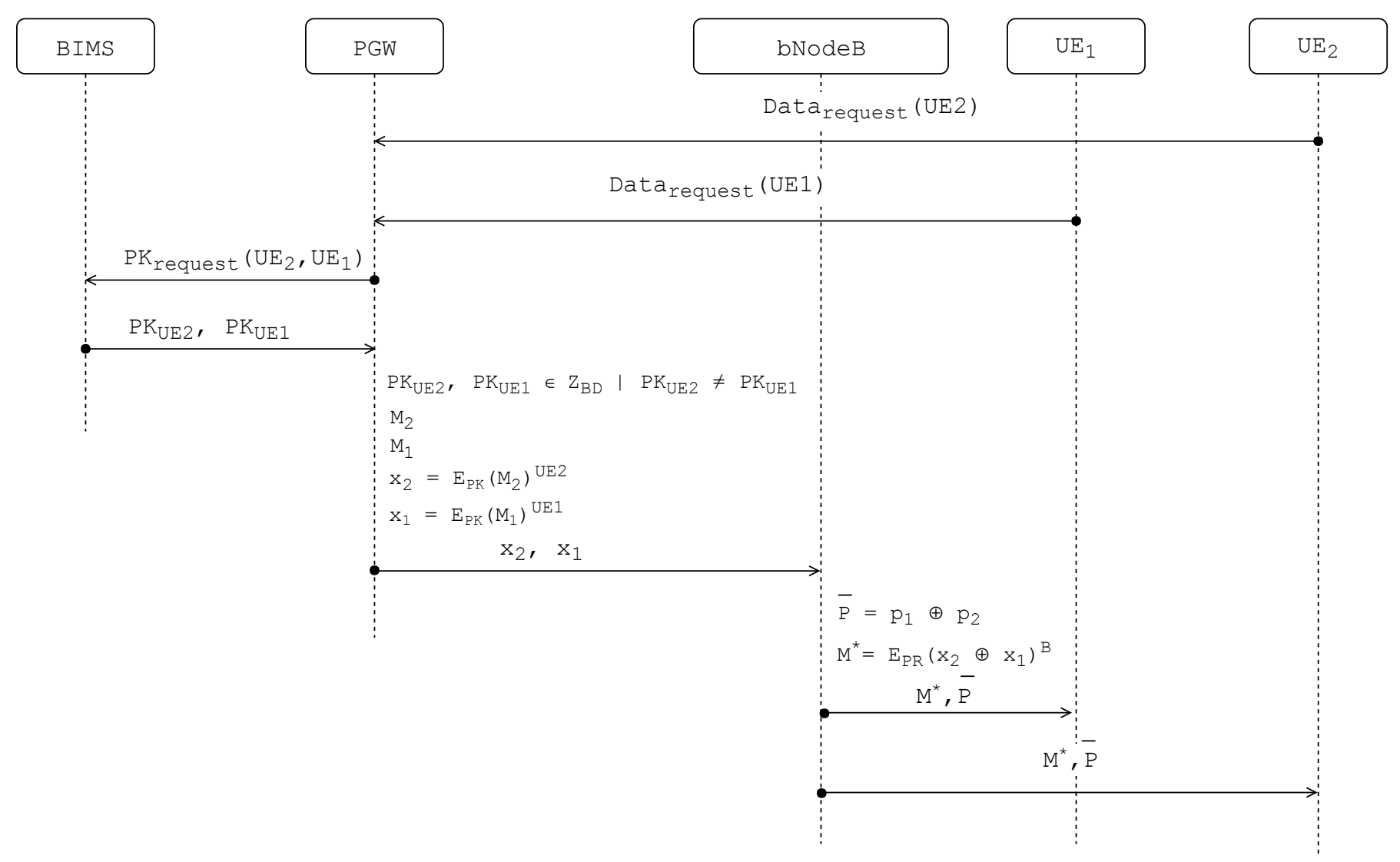

Fig. 3. Proposed data handover scheme.

signals of $\mathrm{UE}_{2}$ in order to extract its own data. After that, it retrieves its own data by computing from $M_{1}$.

5) $\mathrm{UE}_{2}$ extract its data considering signal of $\mathrm{UE}_{1}$ as a noise.

As the existing scheme does not contain any encryption techniques, data becomes vulnerable to attack. Since $U_{1}$ decodes $\mathrm{UE}_{2}$ 's data, $\mathrm{UE}_{1}$ may use $\mathrm{UE}_{2}$ 's data for unethical purposes.

\section{PROPOSED BLOCKCHAIN BASED DATA HANDOVER}

We have proposed a secure data handover process in NOMA with the integration of Blockchain. The proposed scheme contains three parts such as (1) public/private key generation, (2) encryption process in BS, and (3) decryption process in UE.

\section{A. Public/private key generation}

In the proposed scheme, private/public keys play a very significant role. These keys are used for first-phase encryption. The proposed scheme assumes a protected area in UE, named Kaladanda Box (KBox), which preserves credentials like UE's private/public key, BS's private key. An assumption is made that when UE registers in the $\mathrm{BS}, \mathrm{BS}$ shares its private key $P R_{B}$ with every registered UE. $P R_{B}$ uses for second-phase encryption. However, before registering in BS, UE generates the public key and broadcast it for secure communication. In order to construct the public key, a private key is needed. Let $P R_{\mathrm{UE}_{i}}$ is a private key of $i^{t h} \mathrm{UE}$.

$$
P R_{\mathrm{UE}_{i}}=S H A 256\left(I M_{i}, M_{i}, T_{i}, \text { Lat }_{i}, \text { Lon }_{i}\right)
$$

Here, $i \in\{1,2, \ldots, n\}$ and $n$ is total UE and for our proposed scheme $n=2$ and let $\overline{P R}_{\mathrm{UE}}$ is a set of UE's private key.

$$
\overline{P R}_{\mathrm{UE}}=\left\{P R_{\mathrm{UE}_{1}}, P R_{\mathrm{UE}_{2}}, \ldots ., P R_{\mathrm{UE}_{n}}\right\}_{\neq}
$$

Let $G$ is a set of $(x, y)$ coordinates on the elliptic curve.

$$
P K_{\mathrm{UE}_{i}}=P R_{\mathrm{UE}_{i}} \otimes\left(G_{x}, G_{y}\right)
$$

After generating the public key, UE broadcasts, and saves public key KBox for decrypting data bits.

\section{B. Encryption process in $B S$}

In this part, a two-phase encryption is adopted in order to give protection against different kinds of attacks, as shown in Fig. 3. In the first phase, an asymmetric encryption technique is adopted and data is encrypted by UE's public key so that only authorized UE can decrypt it using the private key. In the second phase, a symmetric encryption technique is adopted and data again encrypted using BS's private key so that only legitimate can access data and become safe from 
eavesdroppers. We have assumed a modified version of eNodeB named blockchain supported Node-B (bNodeB) which supports blockchain integration in the BS. First, both $\mathrm{UE}_{2}$ and $\mathrm{UE}_{1}$ send data requests to bNodeB by sending Data $\mathrm{Drequest}\left(\mathrm{UE}_{2}\right)$ and Data request $\left(\mathrm{UE}_{1}\right)$ respectively. bNodeB forwards the request to $\mathrm{PGW}$ to process the request. When PGW gets requests from $\mathrm{UE}_{2}$ and $\mathrm{UE}_{1}, \mathrm{PGW}$ retrieves public keys of both $\mathrm{UE}_{2}$ and $\mathrm{UE}_{1}$ from blockchain based identity management server (BIMS) by sending $\mathrm{PK}_{\text {request }}\left(\mathrm{UE}_{2}, \mathrm{UE}_{1}\right)$. BIMS responds with $\mathrm{PK}_{\mathrm{UE} 2}$ and $\mathrm{PK}_{\mathrm{UE} 1}$. Let $Z_{B D}$ is the blockchain based database that manages public keys.

$$
\mathrm{PK}_{\mathrm{UE} 2}, \mathrm{PK}_{\mathrm{UE} 1} \in Z_{B D} \mid \mathrm{PK}_{\mathrm{UE} 2} \neq \mathrm{PK}_{\mathrm{UE} 1}
$$

As PK is used both as an identity and as securing data packet, each user's PK should be unique. In order to make PK unique, we choose not only unique IMEI, MAC address, but also timestamp and spatial information for generating users' key. The key becomes strong after mixing timestamp and spatial information because if any hacker tries to clone IMEI or spoof the MAC address, he still needs timestamp and spatial information to clone private/public key. However, PGW picks $M_{2}$ and $M_{1}$ for $\mathrm{UE}_{2}$ and $\mathrm{UE}_{1}$ respectively. Let $x_{2}$ and $x_{1}$ are encrypted packets.

$$
\begin{aligned}
& x_{2}=E_{P K}\left(M_{2}\right)^{U E 2} \\
& x_{1}=E_{P K}\left(M_{1}\right)^{U E 1}
\end{aligned}
$$

PGW forwards these encrypted data $x_{2}$ and $x_{1}$ to bNodeB for further processing. As bNodeB receives $x_{2}$ and $x_{1}, \mathrm{bN}$ odeB allocates power $p_{2}$ and $p_{1}$ with signals of $\mathrm{UE}_{2}$ and $\mathrm{UE}_{1}$ respectively, and superposes both signals to $\bar{P}=p_{1} \oplus p_{2}$. After that, both $x_{2}$ and $x_{1}$ experience encryption by employing $P R_{B}$. Let $M^{*}$ is encrypted packets.

$$
M^{*}=E_{P R}\left(x_{2} \oplus x_{1}\right)^{B}
$$

Here, only legitimate users hold $P R_{B}$.

\section{Decryption process in $U E$}

In this part, a two-phase decryption process is discussed in Algorithm 1. Upon receiving a response from bNodeB, both $\mathrm{UE}_{1}$ and $\mathrm{UE}_{2}$ first try to decrypt received packets $D_{P R}\left(M^{*}\right)^{B}$ using $P R_{B}$. As both $\mathrm{UE}_{1}$ and $\mathrm{UE}_{2}$ is legitimate users, both $\mathrm{UE}_{1}$ and $\mathrm{UE}_{2}$ contains $P R_{B}$ in its KBox. After that, $\mathrm{UE}_{1}$ subtracts $\mathrm{UE}_{2}$ 's data after decoding all the bits sequentially in order to extract its own data. As data for both $\mathrm{UE}_{2}$ and $\mathrm{UE}_{1}$ is encrypted by their public key, $\mathrm{UE}_{1}$ requires $\mathrm{UE}_{2}$ 's private key in order to read $\mathrm{UE}_{2}$ 's data. So, $\mathrm{UE}_{2}$ 's data remains protected during decoding. However, $\mathrm{UE}_{1}$ decrypts its data $D_{P R}\left(M_{1}\right)^{\mathrm{UE} 1}$ using $P R_{\mathrm{UE} 1}$. After the decryption process, $\mathrm{UE}_{1}$ retrieves its own data by computing from $\overline{d b m}$. Following this, $\mathrm{UE}_{2}$ decrypts data $D_{P R}\left(M^{*}\right)^{B}$ using $P R_{B}$. UE $\mathrm{UE}_{2}$ extracts its own data considering $\mathrm{UE}_{1}$ 's data as a noise. After extracting data, $\mathrm{UE}_{2}$ decrypts data $D_{P R}\left(M_{2}\right)^{\mathrm{UE} 2}$ using $P R_{\mathrm{UE} 2}$. Finally, $\mathrm{UE}_{2}$ retrieves its own data by computing from $\overline{\mathrm{dbm}}$.

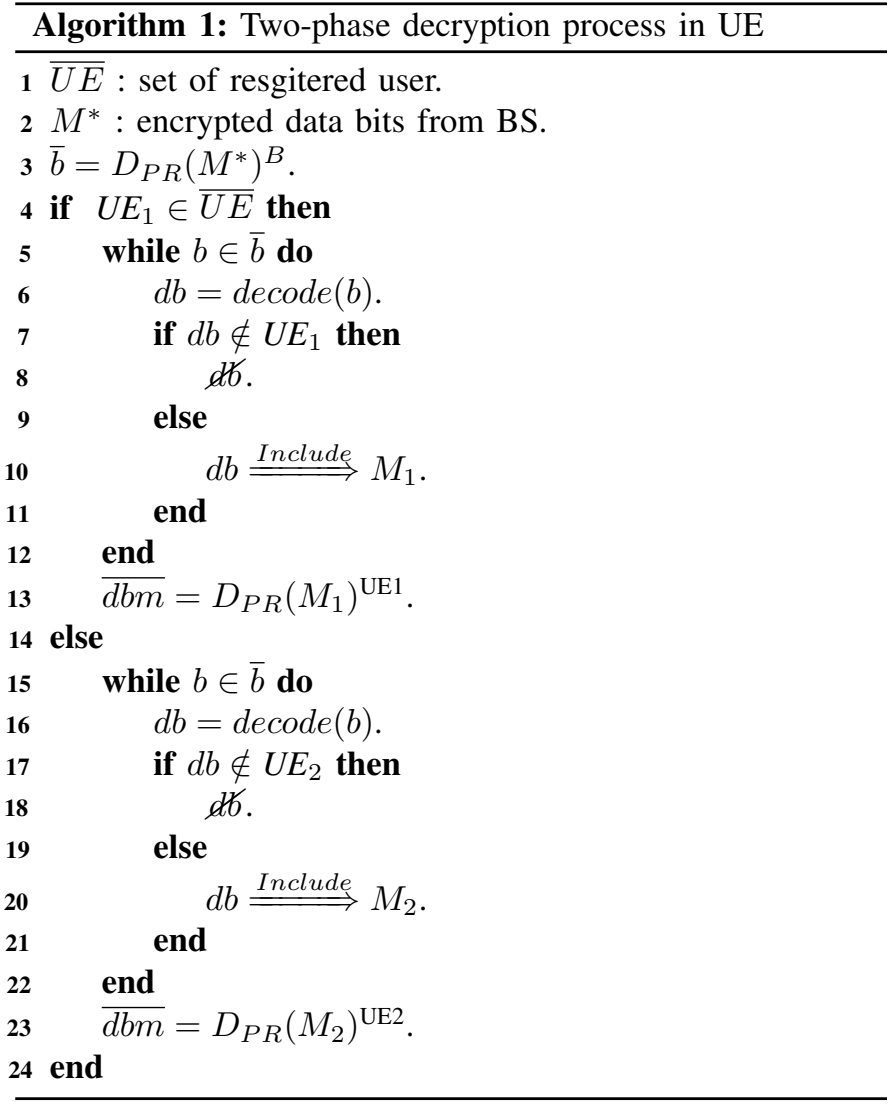

\section{PERformance AnAlysis}

A performance comparison is outlined in Table II The features that take into consideration for performance analysis are delineated below:

User privacy

This feature protects the user personal information from leaking while registering in BS. The proposed scheme has taken into consideration on the issue and proposed to share information in minimum level which is managed in blockchain.

Encryption

This feature protects data from unauthorized access. Both proposed scheme and secure SIC [7] have introduced two-phase encryption. The proposed scheme has adopted asymmetric encryption using the public key in the first phase and symmetric encryption using the private key of $\mathrm{BS}$ in the second phase. On the contrary, secure SIC [7] has used symmetric encryption in both phases.

Key generation

This feature covers comparison of properties that are used for key generation. Secure SIC [7] has utilized IMEI and MAC address for generating the key. On the contrary, the proposed scheme has taken not only IMEI and MAC address into consideration, but also has taken timestamp and spatial information into consideration. The key generation for the attacker 
is much more difficult in the proposed scheme than secure SIC [7].

Protection against spoofing attack

This feature supports the protection against spoofing attack. Both proposed and secure SIC [7] has proposed protection against spoofing attack. However, the solution in secure SIC [7] only covers two vendors named CISCO and Juniper. But the solution in the proposed schemes supports every user.

Protection against data hijacking

This feature supports data protection against unauthorized access. Both the proposed scheme and secure SIC [7] have provided their solution against hijacking data by employing encryption.

TABLE II

Performance COMPaRison BETWEen PROPOSED SCHEME AND SECURE SIC [7]

\begin{tabular}{|c|c|c|}
\hline Proposed & Proposed scheme & Secure SIC [7] \\
\hline User privacy & yes & no \\
\hline Encryption & yes & yes \\
\hline Key generation & $\begin{array}{c}\text { IMEI, MAC } \\
\text { address, } \\
\text { timestamp, spatial } \\
\text { information }\end{array}$ & $\begin{array}{c}\text { IMEI, MAC } \\
\text { address }\end{array}$ \\
\hline $\begin{array}{c}\text { Protection against } \\
\text { spoofing attack }\end{array}$ & for all & $\begin{array}{c}\text { partial (only for } \\
\text { CISCO and } \\
\text { Juniper) }\end{array}$ \\
\hline $\begin{array}{c}\text { Protection against data } \\
\text { hijacking }\end{array}$ & yes & yes \\
\hline
\end{tabular}

\section{CONCLUSION}

In this paper, we have proposed a secure data handover scheme combining with blockchain. In the proposed scheme, UE generates a private key based on IMEI, MAC address, timestamp, Lat, and Lon. After that UE generates public key out of private key and shared with BS and also BS shares its private key to the registered users so that only legitimate users can access transmitted information. Complexity analysis of adopting both symmetric and asymmetric encryption in NOMA along with secrecy analysis is kept for future extension of this paper. However, the detailed discussion regarding UE's identity management using blockchain needs to be researched which can be subjected to future works.

\section{ACKNOWLEDGMENT}

This work was supported by the Brain Korea 21 Plus Project (Department of IT Convergence Engineering, Kumoh National Institute of Technology).

\section{REFERENCES}

[1] S. M. R. Islam, N. Avazov, O. A. Dobre, and K. s. Kwak, "Power-domain non-orthogonal multiple access (NOMA) in 5G systems: Potentials and challenges," IEEE Communications Surveys Tutorials, vol. 19, no. 2, pp. 721-742, Secondquarter 2017.

[2] Z. Ding, M. Peng, and H. V. Poor, "Cooperative non-orthogonal multiple access in 5G systems," IEEE Commun. Lett., vol. 19, no. 8, pp. 14621465, Aug. 2015.
[3] M. F. Kader, M. B. Shahab, and S. Y. Shin, "Exploiting non-orthogonal multiple access in cooperative relay sharing," IEEE Commun. Lett., vol. 21, no. 5, pp. 1159-1162, May 2017.

[4] M. F. Kader and S. Y. Shin, "Cooperative relaying using space-time block coded non-orthogonal multiple access," IEEE Trans. Veh. Technol., vol. 66, no. 7, pp. 5894-5903, Jul. 2017.

[5] M. S. Ali, H. Tabassum, and E. Hossain, "Dynamic user clustering and power allocation for uplink and downlink non-orthogonal multiple access (noma) systems," IEEE Access, vol. 4, pp. 6325-6343, 2016.

[6] Z. Yang, Z. Ding, P. Fan, and N. Al-Dhahir, "A general power allocation scheme to guarantee quality of service in downlink and uplink noma systems," IEEE Transactions on Wireless Communications, vol. 15, no. 11, pp. 7244-7257, Nov 2016.

[7] G. B. Satrya and S. Y. Shin, "Security enhancement to successive interference cancellation algorithm for non-orthogonal multiple access (noma)," in 2017 IEEE 28th Annual International Symposium on Personal, Indoor, and Mobile Radio Communications (PIMRC), Oct 2017, pp. $1-5$.

[8] S. Nakamoto, "Bitcoin: A peer-to-peer electronic cash system," 2008. [Online]. Available: https://bitcoin.org/bitcoin.pdf

[9] X. Li, P. Jiang, T. Chen, X. Luo, and Q. Wen, "A survey on the security of blockchain systems," Future Generation Computer Systems, 2017.

[10] K. Christidis and M. Devetsikiotis, "Blockchains and smart contracts for the internet of things," IEEE Access, vol. 4, pp. 2292-2303, 2016. 